\title{
COHERENT BREMSSTRAHLUNG FROM NEUTRONS
}

\author{
Yuri Kunashenko ${ }^{1}$ \\ Tomsk State Pedagogical University, Tomsk, Russia \\ National Research Tomsk Polytechnic University, Tomsk, Russia
}

The neutron has a spin and anomalous magnetic moment and, therefore, it can interact with electromagnetic field. Schwinger [1] was the first who predicted that fast neutrons can be scattered by atom electric field due to their magnetic moment. The Schwinger scattering of fast neutrons by the atoms was experimentally proved in 1956 [2].

Another mechanism of neutron interaction with electromagnetic field is photon emission from neutrons. For the first time, an emission of the photons from neutrons in an external magnetic field was theoretically studied in [4-6]. This new type of radiation produced at interaction of the anomalous magnetic moment and magnetic field was named "spin" light [6]. Bremsstrahlung and coherent bremsstrahlung from neutrons was considered in [6-8].

It is well known that in addition to the electromagnetic interaction, the neutron takes part in the strong interaction. In the paper [9] it was investigated the contribution of strong interaction of neutron into coherent neutron scattering in the crystals.

In the present report we studied the influence of strong interaction of neutron on coherent bremsstrahlung from neutrons. The results of calculations show that coherent effects exist not only for bremsstrahlung due to electromagnetic Schwinger scattering, but also for bremsstrahlung due to nuclear and interferential scattering of fast neutrons.

\footnotetext{
1 Corresponding author: kunashenko@tpu.ru
} 